\title{
Mutation of SPATA7 in a family with autosomal recessive early-onset retinitis pigmentosa
}

\author{
Chitra Kannabiran ${ }^{\dagger *}$, Lakshmi Palavalli† and Subhadra Jalali ${ }^{\ddagger}$ \\ $\uparrow$ Kallam Anji Reddy Molecular Genetics Laboratory, Prof Brien Holden Eye Research Centre, Hyderabad Eye Research \\ Foundation, L V Prasad Eye Institute, Kallam Anji Reddy Campus, Banjara Hills, Hyderabad 500034, India, $\$$ Srimati \\ Kanuri Santhamma Centre for Vitreoretinal Diseases, L V Prasad Eye Institute, Kallam Anji Reddy Campus, Banjara \\ Hills, Hyderabad 500034, India.
}

*Correspondence to: Chitra Kannabiran, E-mail: chitra@lvpei.org; chitrakannabiran@gmail.com

Received: 09 November 2012; Revised: 11 December 2012; Accepted: 11 December 2012; Published: 12 December 2012

(C) Copyright The Author(s): Published by Library Publishing Media. This is an open access article, published under the terms of the Creative Commons Attribution Non-Commercial License (http://creativecommons.org/licenses/by-nc/2.5). This license permits non-commercial use, distribution and reproduction of the article, provided the original work is appropriately acknowledged with correct citation details.

Retinitis pigmentosa (RP) is a form of hereditary retinal degeneration with a high degree of clinical and genetic heterogeneity. It involves progressive and irreversible degeneration of retinal photoreceptors, manifests as progressive loss of vision with characteristic changes in retinal appearance and may eventually lead to complete blindness. It is inherited in an autosomal dominant, autosomal recessive, $\mathrm{X}$-linked, digenic and mitochondrial modes of inheritance and sometimes shows incomplete penetrance. Autosomal recessive RP (ARRP) has the highest degree of genetic heterogeneity among the various forms with $>35$ genes reported to-date [RetNet database; https://sph.uth.edu/retnet/ sum-dis.htm]. Several genes that have mutations in ARRP are also associated with Leber congenital amaurosis (LCA), a severe retinal dystrophy with congenital onset. Mutations in the SPATA7 gene (spermatogenesis-associated protein 7; MIM 609868) on chromosome 14 are associated with LCA and RP (Wang et al, 2009; Perrault et al, 2010; Mackay et al, 2011). SPATA7 is expressed in the mature mouse retina in the ganglion cell layer, the inner nuclear layer and the inner segments of photoreceptors, human spermatocytes and in the brain (Wang et al, 2009; Perrault et al, 2010), although its function is not understood as yet. To identify the disease gene in Indian families with recessive RP and LCA, we screened for regions of homozygosity in the genome, followed by selection and analysis of candidate genes located within homozygous regions for pathogenic changes.

Affected individuals from 26 families with autosomal recessive RP or LCA were genotyped on SNP arrays containing 6000 SNPs (Illumina Infinium arrays; Illumina Inc, San Diego, CA). Diagnostic criteria and genotyping have been described previously (Kannabiran et al, 2012). SNP genotypes were evaluated for regions of homozygosity common to affected members of each family. Candidate genes mapping to homozygous regions were screened for mutations.
The genes that were screened for sequence changes in the present study included RDH12 and SPATA 7. Primers complementary to flanking intronic regions of each exon were used for PCR amplification of the coding regions of both genes using appropriate conditions. The PCR-amplified products were subjected to bi-directional automated sequencing on the ABI3130XL genetic analyzer (Applied Biosystems Incorporated, Palo Alto, CA) and sequences were compared with reference sequences in the Ensembl Genome Browser (transcript ids ENST00000551171 and ENST00000556553 for RDH12 and SPATA7, respectively). Sequence changes detected in affected individuals were tested for presence in 75 unrelated, normal controls.

Genotyping of 26 families showed a region of homozygosity of over $40 \mathrm{Mb}$ extending from $47 \mathrm{Mb}$ to $91 \mathrm{Mb}$ on chromosome 14 in one family having 2 affected offspring of a consanguineous marriage (pedigree in Figure 1). In this family, we detected 5 homozygous segments shared by both affected siblings, ranging in size from $5 \mathrm{Mb}$ $46 \mathrm{Mb}$. The $46 \mathrm{Mb}$ region of homozygosity extended from 47 to $93 \mathrm{Mb}$ on chromosome 14 and included two known retinal dystrophy genes- the $R D H 12$ (retinol dehydrogenase 12) gene mapping to $68.1-68.2 \mathrm{Mb}$ and the SPATA7 (LCA3; spermatogenesis associated 7) gene, mapping to 88.8-88.9Mb. Screening of coding regions of both genes by PCR amplification and sequencing revealed a frameshifting mutation in SPATA7, consisting of a single base deletion of $\mathrm{C}$ in codon 181 (c.544delC; pGln181fs; data reviewed but not shown), homozygous in both affected siblings. This change is predicted to change the glutamine (Gln) codon at position 181 to serine and terminate at the next codon. It co-segregated with disease in 8 members of the family tested (Figure 1) and was not found in 75 ethnically matched, unrelated normal controls. No variations were detectable in $\mathrm{RDH} 12$. 


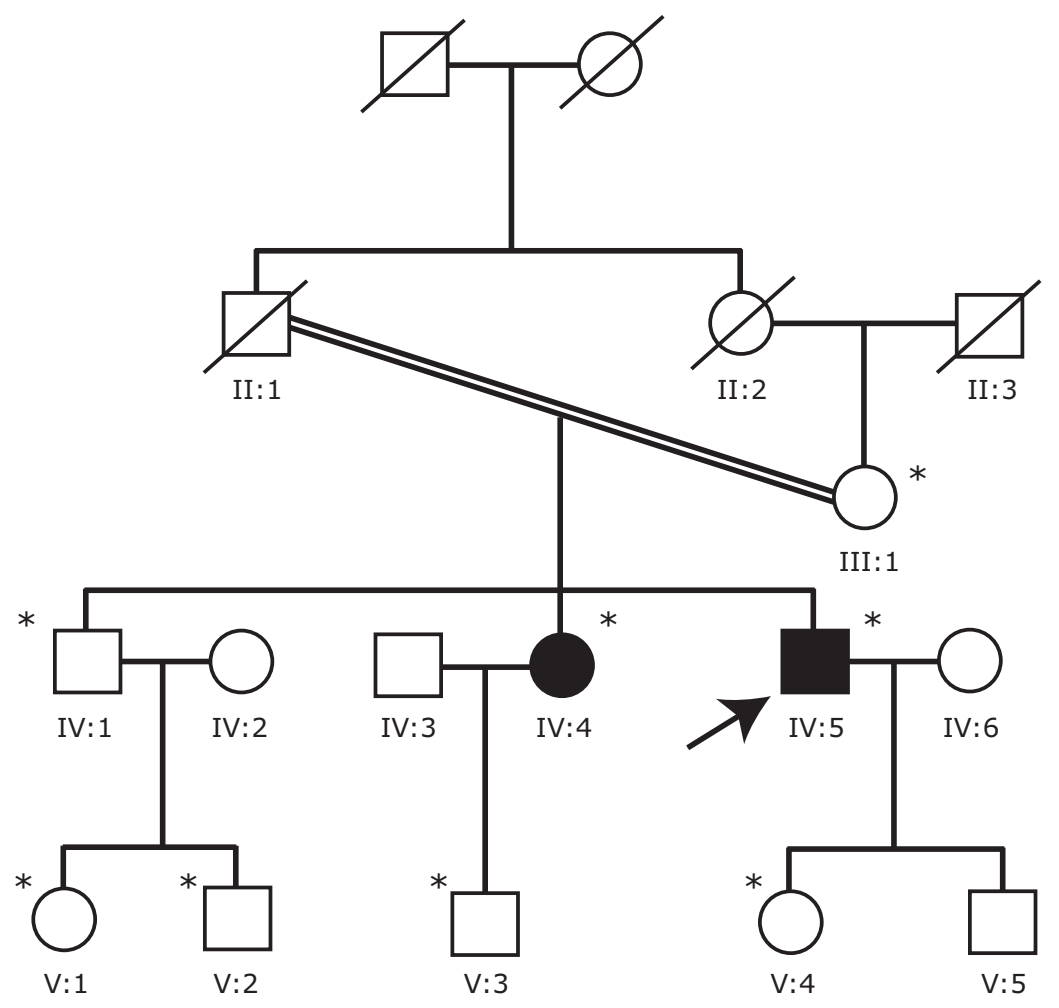

Figure 1. Pedigree of the family screened for SPATA7 mutation. Individuals available for the study are marked with asterisks. Shaded symbols denote affected and clear symbols denote unaffected individuals. Slashes across symbols denote deceased individuals.

The affected individuals in this family reportedly had vision loss and night blindness since early childhood. The proband (IV:5 in Figure 1) was seen at our institution at 37 yrs of age, with best-corrected visual acuities of 20/200 and 20/125 in the right and left eyes respectively. He had horizontal nystagmus in both eyes, a normal anterior segment, clear lens and sluggishly reacting pupils. The fundus showed arterial narrowing, pallor of the optic disc, diffuse and widespread degeneration of the retinal pigment epithelium, and bone spicule-like intraretinal pigment migration (Figure 2). Electroretinography showed extinguished rod and cone responses. He had central visual fields of $<15$ degrees. Over 8 years of follow up, the visual acuity reduced to 20/400 in both eyes and he developed early posterior subcapsular cataracts. His sister (IV:4 in Figure 1) was similarly affected since childhood with a diagnosis of RP and examined in our institution at age 52 years. She had bestcorrected visual acuities of 20/80 and 20/800 in her right and left eyes, respectively, and similar changes in the fundus as the proband.

A genome-wide screen for homozygosity combined with candidate gene evaluation within homozygous regions resulted in the identification of a pathogenic mutation that co-segregated with disease in the family, was absent in the control population and is predicted to lead to a frameshift in the coding sequence of the SPATA7 gene in one out of 26 families with recessive RP or LCA screened in our study. Based on history, patients in this family had an onset of retinal dystrophy in childhood. SPATA7 mutations have been associated with early-onset RP or LCA in previous

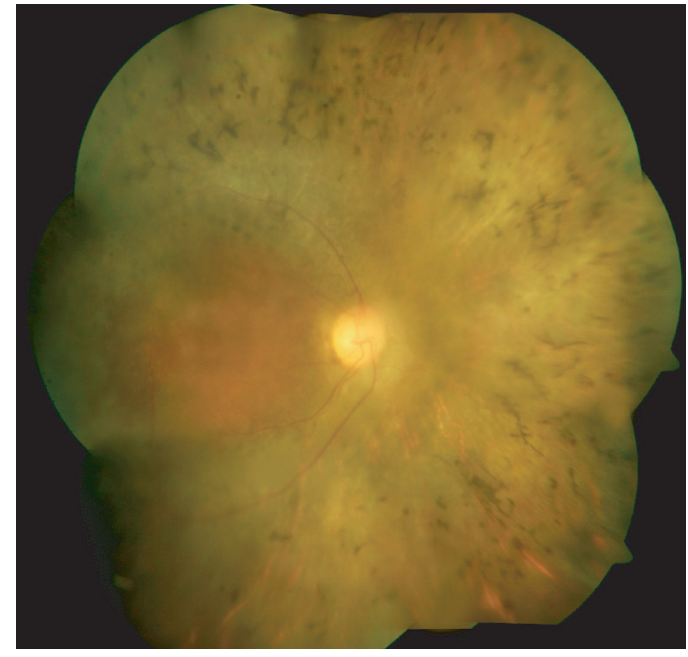

Figure 2. Fundus photomontage of right eye of proband showing typical features of bilateral retinitis pigmentosa including disc pallor, peripheral bone corpuscular pigment migration, severe arterial attenuation, vitreous changes causing grade 1 haze in media, and diffuse retinal pigment epithelial (RPE) degeneration giving a grayish hue to the retina with relative sparing of central macular area.

studies and clinical manifestations include nystagmus, nyctalopia, severe constriction of visual fields, and widespread RPE atrophy with little or no pigmentary deposits, vascular attenuation and minimal disc pallor (Wang et al, 2009; Perrault et al, 2010; Mackay et al, 2011). The majority 
of mutations identified so far are nonsense, frameshift or splice mutations. Phenotypic variability has also been documented, with age at onset and rate of progression of the disease varying between individuals with different mutations in SPATA7 (Avila-Fernandez et al, 2011). Further, variable phenotypes were noted between patients with the same mutation (pR85X), which was found associated with a typical form of RP that had its onset in the $2^{\text {nd }}-3^{\text {rd }}$ decades, as well as with LCA, with onset at birth (Avila-Fernandez et al, 2011; Mackay et al, 2011).

We identified a pathogenic mutation in SPATA7 in a consanguineous family with early-onset RP, a genetically highly heterogeneous disorder. These data suggest a range of clinical phenotypes arising from SPATA7 mutations with common features being severe and progressive phenotypes.

\section{ABBREVIATIONS}

$\mathrm{RP}$; retinitis pigmentosa

ARRP; autosomal recessive retinitis pigmentosa

LCA; Leber congenital amaurosis

SNP; single nucleotide polymorphism

SPATA7; spermatogenesis-associated protein 7

\section{COMPETING INTERESTS}

None declared.

\section{ACKNOWLEDGEMENTS}

This work was supported by Program Support from the Dept of Biotechnology Govt of India, and by the Champalimaud Foundation, Portugal.

\section{REFERENCES}

Avila-Fernandez A, Corton M, Lopez-Molina MI et al. 2011. Lateonset retinitis pigmentosa. Ophthalmology, 118, 2523-2524.

Kannabiran C, Singh H, Sahini N et al. 2012. Mutations in TULP1, NR2E3, and MFRP genes in Indian families with autosomal recessive retinitis pigmentosa. Mol Vis, 18, 1165-1174.

Mackay DS, Ocaka LA, Borman AD et al. 2011. Screening of SPATA7 in patients with Leber congenital amaurosis and severe childhood-onset retinal dystrophy reveals disease-causing mutations. Invest Ophthalmol Vis Sci, 52, 3032-3038.

Perrault I, Hanein S, Gerard X et al. 2010. Spectrum of SPATA7 mutations in Leber congenital amaurosis and delineation of the associated phenotype. Hum Mutat, Mutation in Brief 31, E1241-E1250.

Wang H, den Hollander AI, Maoyedi Y et al. 2009. Mutations in SPATA7 cause Leber congenital amaurosis and juvenile retinitis pigmentosa. Am J Hum Genet, 84, 380-387. 